MS4-O3 Pitfalls of crystallographic reasoning, and linking crystallographic model and data quality

Kay Diederichs ${ }^{1}$

1. University of Konstanz, D-78457 Konstanz, Germany

email: kay.diederichs@uni-konstanz.de

In the last decades, macromolecular crystallography has been extremely successful. But all that glitters is not gold: some of the concepts developed decades ago now need revision.

For example, analysis of crystallographic data quality is still somewhat fixated on values of $\mathrm{R}$ (also called $\mathrm{R}_{\mathrm{sym}}$ ), and the distinction between accuracy and precision, as well as indicators of unmerged data precision and those of merged data precision still has to be realized in the crystallographic community. Furthermore, logical flaws and oversimplifications lead to inappropriate shortcurs that often reduce the quality of structures.

Recently we pointed out (Karplus and Diederichs [2012] Science 336, 1030) that the high-resolution value of $\mathrm{R}_{\text {merge }}$ is not related to $\mathrm{R}_{\text {work }} / \mathrm{R}_{\text {free }}$ of a crystallographic model, andis therefore work unsuitable to define a high-resolution cutoff of data to be included in refinement. A more meaningful indicator $\left(\mathrm{CC}_{1 / 2}\right)$ has been suggested and a derived quantity $\left(\mathrm{CC}^{*}\right)$ was shown to be limiting for $\mathrm{CC}_{\text {work }} / \mathrm{CC}_{\text {free }}$ of a crystallographic model.

This talk will demonstrate that as a consequence, crystallographers are now in a position to better understand the properties of their data, which may be used to obtain more accurate crystallographic models.

Keywords: refinement, high-resolution cutoff, precision, accuracy, data quality indicators

\section{MS4-O4 Advances in synchrotron data collection protocols for experimental phasing}

Aaron D. Finke ${ }^{1}$, Tobias Weinert ${ }^{1}$, Ezequiel Panepucci ${ }^{1}$, Claus Fensburg $^{2}$, Clemens Vonrhein ${ }^{2}$, Gerard Bricogne ${ }^{2}$, Vincent Olieric $^{1}$, Meitian Wang ${ }^{1}$

1. Swiss Light Source, Paul Scherrer Institute, CH-5232 Villigen, Switzerland

2. Global Phasing Ltd., Sheraton House, Castle Park, Cambridge CB3 0AX, United Kingdom

email: aaron.finke@psi.ch

Experimental phasing by single- or multiple-wavelength anomalous diffraction (SAD or $\mathrm{MAD})$ is the most popular method of de novo macromolecular structure determination. Recent overhauls at third-generation synchrotron light sources have dramatically sped up phasing data collection protocols. In particular, the reintroduction of multi-axis goniometers has enabled two major advances in anomalous data collection: the ability to collect "true" high-multiplicity, low-dose datasets on a single crystal entity, maximizing the anomalous signal output while minimizing both systematic and random measurement errors; and alignment of even-fold crystal symmetry axes along the spindle axis to collect Bijvoet pairs, thus reducing radiation damage-induced errors in measurement. We present our results in applying both strategies to challenging problems in experimental phasing.

Keywords: experimental phasing, SAD, MAD, multi-axis goniometer 